\title{
Minimally Invasive/Percutaneous Talus Fracture Osteosynthesis - A Case Report.
}

\author{
Dr.Mazharuddin Ali Khan ${ }^{1}$, Dr.Chinnala Srujan Kumar ${ }^{2}$, \\ Dr. Mohammed Amanulhaq Qureshi ${ }^{3}$ And Dr.Sarosh Haidry ${ }^{4}$ \\ ${ }^{I}$ Department Of Orthopaedics, Professor And Head Of The Department, Deccan College Of Medical Sciences. \\ ${ }^{2}$ Department Of Orthopaedics, Senior-Resident, Deccan College Of Medical Sciences. \\ ${ }^{3}$ Department Of Orthopaedics, Post-Graduate, Deccan College Of Medical Sciences. \\ ${ }^{4}$ Department Of Orthopaedics, Post-Graduate, Deccan College Of Medical Sciences.
}

\begin{abstract}
The talus is involved in about 2\% of all lower extremity injuries and about 5-7\% of foot injuries. Fractures of Talus are relatively uncommon and can be associated with significant complications. Because it articulates with important joints i.e., ankle, subtalar, and talonavicular, it is one of the most important bones to stabilize and mobilize soon after a foot injury. Talar fractures are usually associated with hyperdorsiflexion of the ankle as the talar neck impacts the anterior margin of the tibia, such as in a motor vehicle accident or fall from height. We describe the case of a 21year old male presenting with displaced talus fracture of right leg which was treated with a minimally invasive technique.
\end{abstract}

Keywords: Talus Neck fracture, Minimally invasive technique, limited complications, less hospitalization period.

\section{Introduction}

The second largest tarsal Bone is Talus, with more than half of its surface covered by articular cartilage. It has no muscular or tendinous attachments but has a groove for the flexor hallicus longus tendon and is supported solely by the joint capsules, ligaments, and synovial tissues. The talus articulates superiorly with the tibia and fibula in the ankle mortise and the calcaneus and navicular inferiorly. Body weight is transmitted through the tibia directly to the superior surface of the talus. The anterior portion of the body is wider than the posterior portion, giving stability to the ankle. The neck of the talus is the most vulnerable region to fracture and connects to the head, which connects to the navicular and calcaneous and articulates with the spring ligament inferiorly along the distal medial aspect, the sustentaculum tali along the medial inferior aspect, and the deltoid ligament direct medially at the level of the ankle. The major blood supply to the body is from the artery of the tarsal canal (posterior tibial artery). Blood is also supplied by arteries to the sinus tarsi (peroneal and dorsalis pedis arteries); the deltoid artery (posterior tibial artery) which supplies the medial body and the superior neck vessels (anterior tibial artery)[1,2,3](Fig.1).

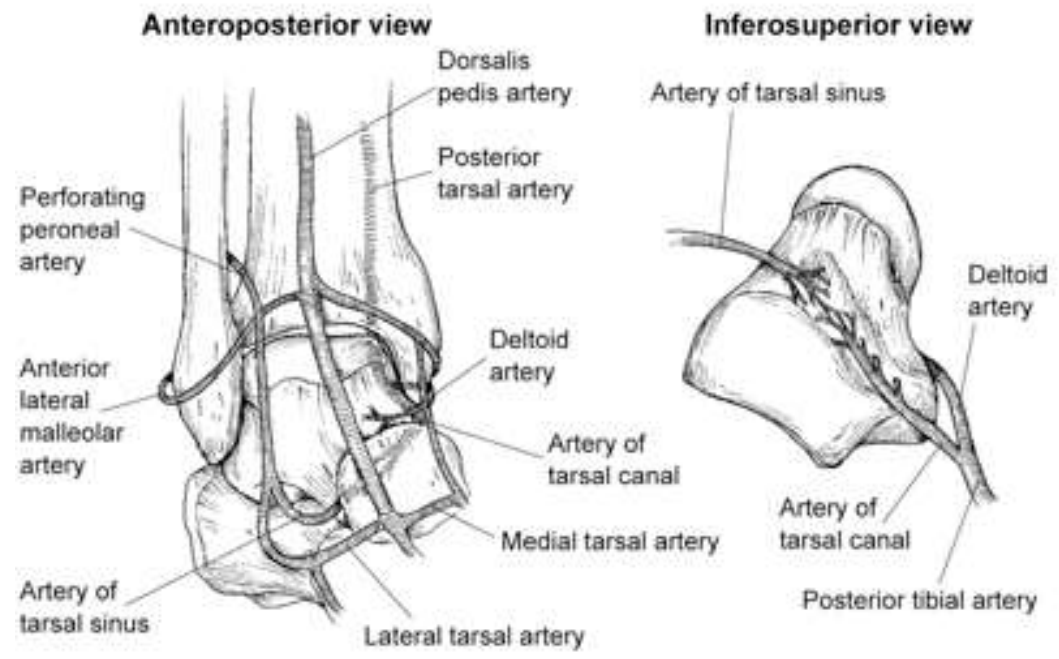

Figure 1: Blood supply of Talus. 
The most commonly used classification system is the Hawkins classification of talar neck fractures[4].

Type I : Nondisplaced talar neck fracture

Type II : Displaced with subluxation or dislocation of subtalar joint

Type III : Displaced with dislocation of body from ankle mortise

Type IV : Displaced with subluxation or dislocation of talonavicular joint

Other types of fractures include talar head fractures, talar body fractures, lateral process fractures, and posterior process fractures.

\section{Case Presentation}

A 21-year-old male patient presented to the emergency department with pain and swelling in the right ankle with inability to bear weight over his right leg following a road traffic accident. On local examination there was a gross swelling over right ankle with no external injuries, with tenderness at the talar region and restricted range of ankle movements. Both anteroposterior and lateral radiographs revealed a displaced Hawkins type II talus fracture with incongruity of tibio-talar joint (Fig.2a,b).

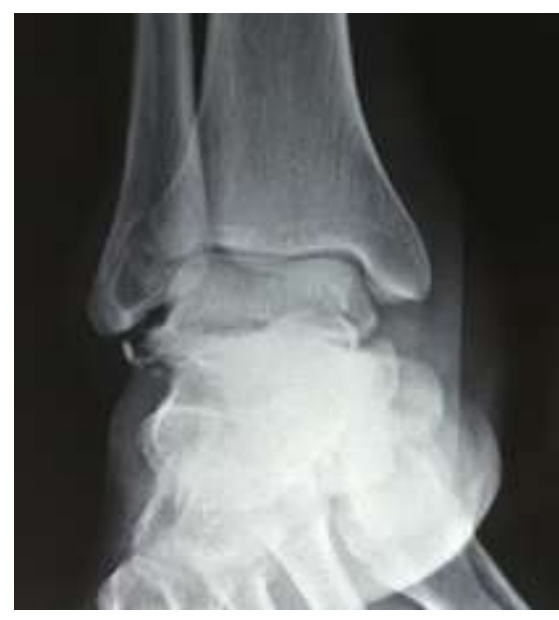

Figure 2a: Plain Radiograph AP view showing fracture neck of Talus with Lateral displacement(Hawkins

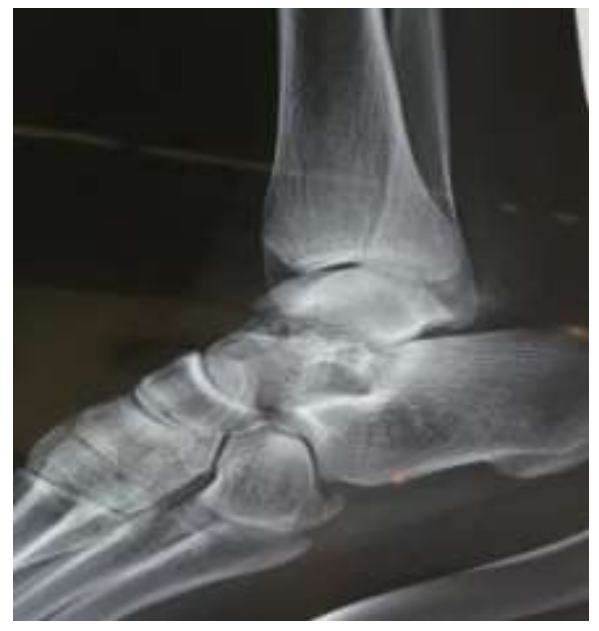

Figure 2b: Plain Radiograph lateral view showing Fracture neck of

Rest of the routine blood investigations were normal. While an open reduction and internal fixation is recommended for such type of fractures, which takes a lot of time for healing, with risk of infections, later need for re-surgery for implant removal and prolonged period of loss of working hours, in view of all the above complications we planned for a minimally invasive procedure. Two pairs of Kirschner wires, one inferomedially and another pair infero-laterally were inserted form dorsum of foot passing from talar head medially and laterally crossing the fracture site into the body of the talus in a converging fashion to provide more rigid and stable fracture fixation, without disturbing the articular surfaces under C-arm guidance after manual reduction(Fig.3a,b) Patient was immobilized with a below knee cast for about 4 week and was then mobilized once the Kirschner wires were removed. Ankle range of movements was upto near normal and satisfactory to us and to the patient as well. 


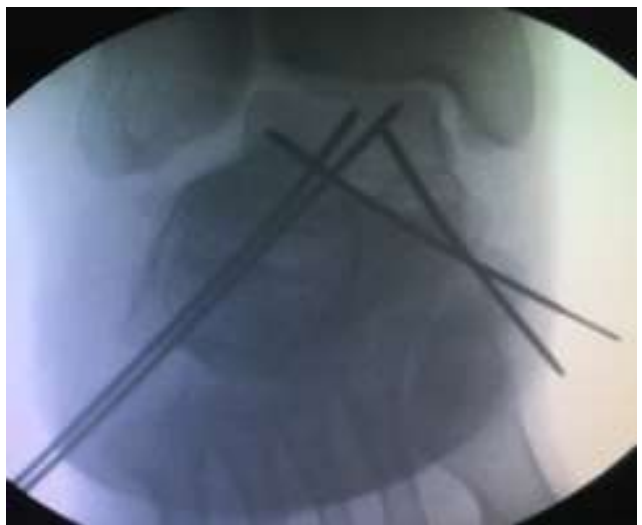

Figure 3a: Intra-operative Fracture fixation with Kirschner wires under C-Arm guidance. AP View.

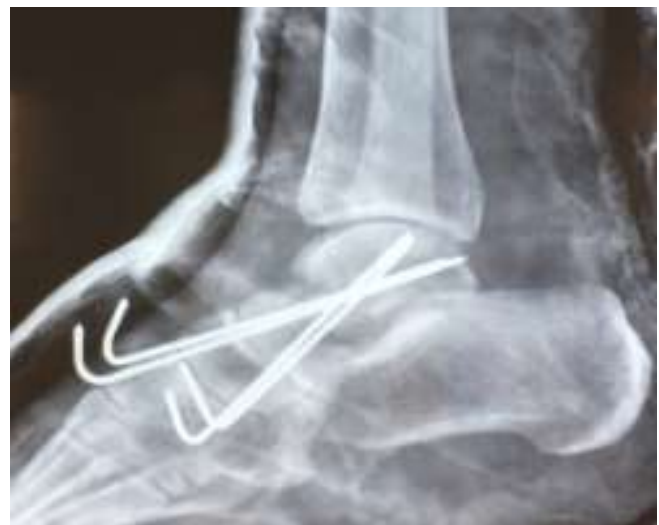

Figure 3b: Post-operative plain radiograph with Kirschner wires insitu, in Lateral View.

\section{Discussion}

Approximately $50 \%$ of all talar fractures occur in the talar neck region, these occur when the narrow neck of the talus, with its less dense trabecular bone, strikes the stronger anterior tibial crest. Type II fractures are a combination of fracture of the talar neck with subluxation or dislocation of the subtalar joint. Talus fracture may be only part of the total spectrum of the patient's injuries, and a general trauma survey should be a part of evaluation in every patient. Special attention should also be directed to the thoracolumbar spine, as spine fractures have been found to be associated with talar neck and body fractures. Management of Hawkins Type I fracture involves a short leg cast or boot for 8-12 weeks and no weight-bearing for 6 weeks, Hawkins Type II, III and IV fractures almost and always open reduction and internal fixation (ORIF) is recommended. In lateral process fracture if there is a displacement of less than $2 \mathrm{~mm}$, use of a short leg cast or boot for 6 weeks with non weight bearing for 4 weeks is advised and if there is more than $2 \mathrm{~mm}$ displacement, ORIF is recommended. Treatment of posterior process fracture is also determined by displacement. A non-displaced or minimally displaced posterior process fractures are treated with a short leg cast for 6 weeks with non weight bearing for 4 weeks. For displaced fractures, ORIF is recommended. Nondisplaced talar head fractures are treated with a short leg cast molded to preserve the longitudinal arch; partial weight-bearing is recommended for 6 weeks. ORIF is necessary for displaced talar head fractures[5]. The prognosis for talus fractures is related to the degree of damage to its blood supply and the damage to the articular surfaces. Complications are related to the degree of displacement as well as risk of avascular necrosis. One of the most common complications of talar neck fractures is avascular necrosis due to injury to the artery of the tarsal canal, which supplies the body, and branches of the dorsalis pedis and peroneal arteries, which supply the head and neck.

Risk of avascular necrosis is stratified according to the Hawkins classification[1]:

Hawkins Type I: $0-15 \%$

Hawkins Type II: $20-50 \%$

Hawkins Type III 20-100\%

Hawkins Type IV: $100 \%$

Open fractures can occur in $15-25 \%$ of injuries, reflecting the high-energy mechanism of injury. The infection rate is up to $40 \%$ in open talus fractures. Post-traumatic arthritis occurs in 40-90\% of cases. Delayed union or nonunion occurs in approximately $15 \%$ of cases, and malunion is also a concern. Other complications include skin slough secondary to prolonged dislocation, interposition of the long flexor tendons, and foot compartment syndrome. The goal of treatment of talar neck fractures is anatomic reduction, which requires proper attention towards rotation, length, and angulation of the neck. Biomechanical studies on cadavers have shown why precisely reducing talar neck fractures leads to better outcomes. In one cadaveric study, displacements by as little as $2 \mathrm{~mm}$ were found to alter the contact characteristics of the subtalar joint, with dorsal and medial or varus displacement causing the greatest change. The weight-bearing load pathway changed, and contact stress was decreased in the anterior and middle facets but was more localized in the posterior facet[6]. In another study, varus alignment was created by removing a medially based wedge of bone from the talar neck. This resulted in inability to evert the hindfoot, and the altered foot position was characterized by internal rotation of the calcaneus, heel varus, and forefoot adduction[7]. The altered hindfoot mechanics with a talar neck fracture may be one factor that leads to subtalar posttraumatic arthrosis. For these reasons, open reduction and internal fixation is recommended for displaced fractures. 


\section{Management of Type II Fractures[1]}

Initial management of displaced talar neck fractures should involve prompt reduction to minimize soft tissue compromise. This can often be performed in the emergency room. However, repeated forceful reduction attempts should be avoided. The foot is plantar-flexed, bringing the head in line with the body. The heel can then be manipulated into either inversion or eversion, depending on whether the subtalar component of the displacement is medial or lateral. Anatomic reduction of this fracture is difficult to obtain by closed means. Rotational alignment of the talar neck is very difficult to judge on plain radiographs. Even minimal residual displacement can adversely affect subtalar joint mechanics and is therefore unacceptable[6]. Even if closed reduction is successful in obtaining an anatomic reduction, immobilization in significant plantar-flexion is typically necessary to maintain position. For these reasons, operative treatment of all type II fractures has been recommended.

\section{Conclusion}

ORIF results in lower rates of nonunion, shorter time to union, earlier weight-bearing, better anatomical reduction, and lower rate of avascular necrosis than closed treatment. But this case has been presented with similar outcome treated with minimally invasive procedure with much shorter hospital stay, early weight bearing and with no requirement of re-surgery for implant removal unlike ORIF. However outcome depends on accuracy of reduction, avascular necrosis, and degree of joint injury.

\section{Conflicts of Intrest : None.}

\section{Referances}

[1]. Paul T. Fortin, MD, and Jeffrey E. Balazsy, MD: Talus Fractures: Evaluation and Treatment. J Am Acad Orthop Surg 2001;9:114127.

[2]. Haliburton RA, Sullivan CR, Kelly PJ, Peterson LFA: The extra-osseous and intra-osseous blood supply of the talus. J Bone Joint Surg Am 1958;40: 1115-1120.

[3]. Mulfinger GL, Trueta J: The blood supply of the talus. J Bone Joint Surg Br 1970;52:160-167.

[4]. Hawkins LG: Fractures of the neck of the talus. J Bone Joint Surg Am 1970;52: 991-1002.

[5]. Talar fractures, OrthopaedicsOne Articles, Page 301 of 372.

[6]. Sangeorzan BJ, Wagner UA, Harrington RM, Tencer AF: Contact characteristics of the subtalar joint: The effect of talar neck misalignment. J Orthop Res 1992;10:544-551.

[7]. Daniels TR, Smith JW, Ross TI: Varus malalignment of the talar neck: Its effect on the position of the foot and on subtalar motion. J Bone Joint Surg Am 1996;78:1559-1567. 